\title{
S\&P 500 Forecasting by Fuzzy Neural Network
}

\author{
Shang-Jen Chuang and Chung-Yung Lee
}

\begin{abstract}
In order to establish a new method to predict the highest-price, lowest-price, and the close-price in the daily $S \& P$ 500 Index, this research use a combination of Back -propagation Neural Network (BPNN) and Fuzzy Controller as the tools to build up models. The data used in this research were collected between 03. Jan. 2000 to 02 . Nov. 2012, 3230 days in total. The neural network input variables include daily open-price, a day before, two days before, and a week prior's open-price, close-price, highest-price, lowest price, and the trading volume. The data collected from 2000 2007 were used for training; while those from 2008 2012 were used for testing.
\end{abstract}

Index Terms-Fuzzy neural network, back-propagation neural network, fuzzy controller, stock forecast.

\section{INTRODUCTION}

On account of the technology improvement, the operation ability of computer has been largely promoted, and now has the ability to deal with great amount of complex information problems. Neural Network is a branch of artificial intelligence; due to its' good fault tolerant ability, it can even make precise prediction on noise. It has been widely used in stock market, futures contract, and bond's prediction and analysis. According to previous researches, it has been proved that Neural Network has reached a certain extent on reliability and fault tolerant ability while dealing with complex information. [1]

Stock market prediction is not an easy job, due to lots of factors. From 1980, a lot of researchers used BPNN to predict the fluctuations in stock market. Yet BPNN has its' limitation, including lower learning speed, lower learning efficiency, and will enter partial minimum amount. [2]

A combination of BPNN and Fuzzy Controller was used in this paper, in order to make prediction on the highest-price, lowest-price, and the close-price in the daily S\&P 500 Index. A comparison between BPNN and the combination of BPNN and Fuzzy Controller's prediction ability were also made in this research.

\section{BaCk-Propagation NeURAL NeTwork, FuZZY}

BPNN can be split into two parts: learning, and testing. It's learning progress were called supervised learning, which will gather training data and target data from the historical data. Then input training data into the network, try to let error function get the minimum deviation (expected value minus the output value), then let the deviation back to the network

Manuscript received July 25, 2013; revised September 25, 2013.

The authors are with the Electronic Communication Engineering, National Kaohsiung Marine University, Kaohsiung, Taiwan (e-mail: david@mail.nkmu.edu.tw, 1011536101@stu.nkmu.edu.tw). from output layer to input layer, by using the gradient steepest descent method. [3]

The struck of the network (see Fig. 1) contains:

1) Input layer: In the input layer we used 17 neuroses in this research.

2) Hidden layer: The number of neuroses in this layer is not to get a standard presentation method, researchers often use try-error method to get the appropriate numbers. In trans function, we used Sigmoidal function.

3) Output layer: 3 neuroses were used in this layer.



Fig. 1. Back-propagation neural network model.

\section{THE STRUCTURE OF THE FUZZY CONTROLLER}

Fuzzy System has been widely used in Automatic control, pattern recognition, decision analysis, and Time serial Signal Processing...etc. The basic framework (see Fig. 2) and functions of the Fuzzy Controller are as follows: [4]

1) Fuzzification

2) Fuzzy Rule Base

3) Fuzzy Inference Mechanism

4) Defuzzifierion



Fig. 2. Fuzzy controller composition diagram.

\section{A. Design of Fuzzy Controller}

The Fuzzy Input Membership function used in this research were Mean Absolute Percentage Error (MAPE) (see Fig. 3), and the deviation between the present and former MAPE (see Fig. 4).

Output membership function were learning rate $(\eta)$ and the momentum rate( $\alpha$ ), (see Fig. 5) The Fuzzy rules graph were shown in (see Table I) In this research, the fuzzy language for 
input and output are as follows: [5]

1) NB: Negative Big

2) NS: Negative Small

3) ZE: Zero

4) PS: Positive Small

5) PB: Positive Big

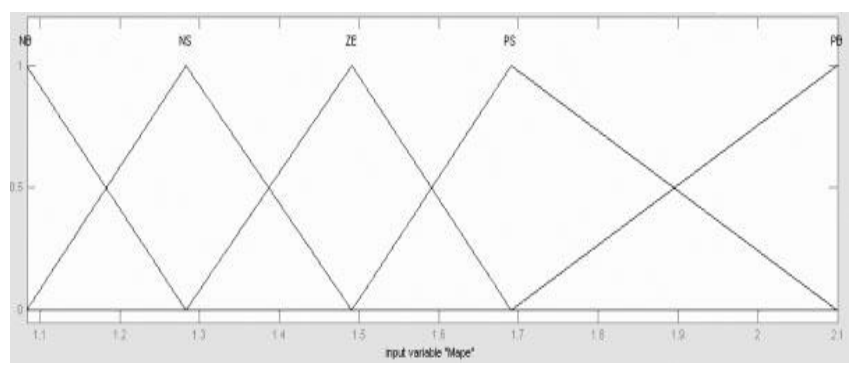

Fig. 3. Membership function for e.

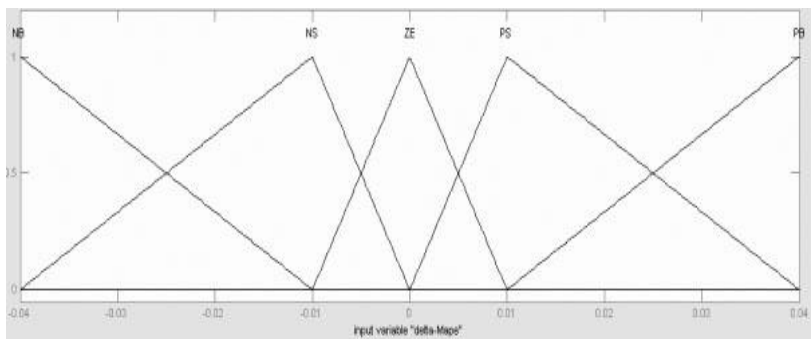

Fig. 4. Membership function for $\Delta \mathrm{e}$.

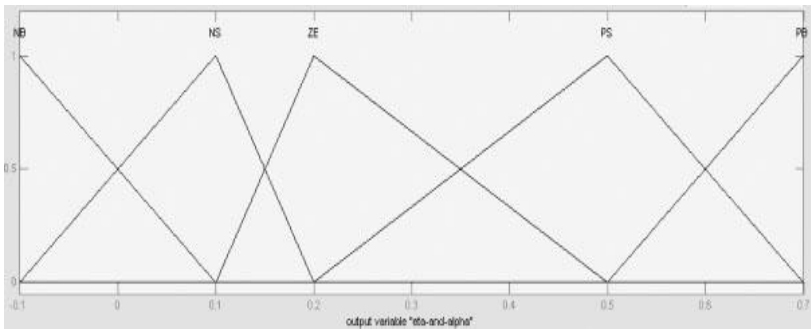

Fig. 5. Membership function for $\eta$ and $\alpha$.

TABLE I: DECISION BASE FOR FUZZY LOGIC CONTROL OF H AND A

\begin{tabular}{|c|c|c|c|c|c|}
\hline $\mathbf{e}$ & NE & NS & ZE & PS & PB \\
\hline NB & NS & PS & PS & PB & PB \\
\hline NS & NB & NS & ZE & PS & PB \\
\hline ZE & NB & NB & NS & ZE & PB \\
\hline PS & NB & NB & NB & NB & NB \\
\hline PB & NS & ZE & PS & PB & PB \\
\hline
\end{tabular}

\section{SySTEM STRUCTURE}

\section{A. Fuzzy Neural Network Structure}

In this research, BPNN and Fuzzy Controller were combined, in order to use this new neural network model to predict stock market. The frame work of this model (see Fig. 6) shows that after BPNN complete its' first training, it will compare this time's MAPE with the MAPE got from last time; then it will do a fuzzy inference. After that, it will alter the learning rate $(\eta)$, and the momentum rate $(\alpha)$ before carry on to the next training cycle.

By using this method, the BPNN can automatically adjust to better learning rate and momentum rate, and lead to better effects and outcomes.

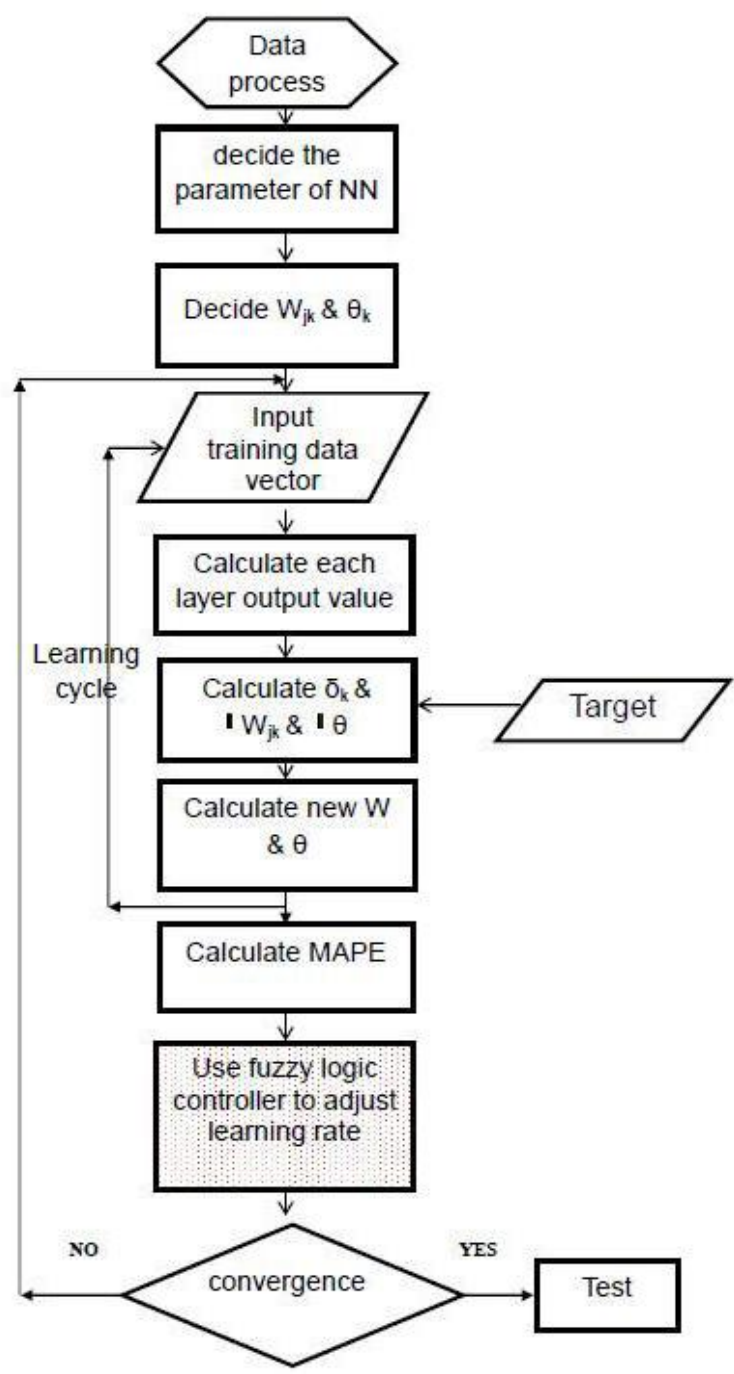

Fig. 6. Fuzzy neural network flowchart.

\section{B. Stock Price Acquisition and Analysis}

The data in this paper were gathered from S\&P 500 Index, and used 17 dimension data to analyse the daily price, a day before, two days before, and a week prior's open-price, close-price, highest-price, lowest price, and the trading volume, in order to predict the daily highest-price, lowest-price, and the close-price. [6], [7]

\section{EXPERIMENTAL RESULTS}

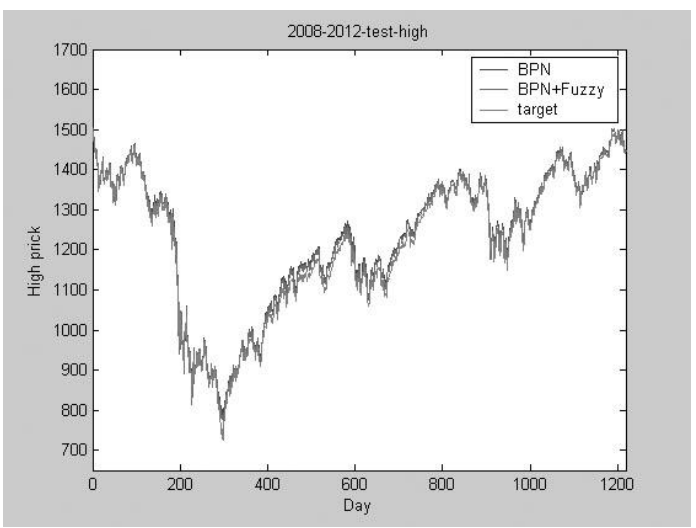

Fig. 7. 2008-2012 actual and predicted values comparison chart - the highest price. 
The S\&P 500 Index used in this paper were collected between $3^{\text {rd }}$, Jan, 2000 till $2^{\text {nd }}$, Nov, 2012, and categorized those data into training data and testing data., both used daily data as input variables. Two kinds of Neural Network Models were used to predict and then compare the results: BPNN, and a combination of BPNN and Fuzzy Controller. The comparison results were shown below (see Table II); while the comparison between highest-price, lowest -price, and the close price showed that the combination of BPNN and Fuzzy Controller's prediction value were closer to the target value (see Fig. 7-Fig. 9).

TABLE II: COMPARISON OF THE RESULTS OF TRAINING AND TESTING

\begin{tabular}{|c|c|c|c|}
\hline \multicolumn{2}{|c|}{} & $\begin{array}{c}\text { 2000-2007 year } \\
\text { MAPE for Training }\end{array}$ & $\begin{array}{c}\text { 2008-2012 year } \\
\text { MAPE for Test }\end{array}$ \\
\hline \multirow{4}{*}{ BPN+Fuzzy } & High & 0.462 & 1.218 \\
\cline { 2 - 4 } & Low & 0.549 & 1.270 \\
\cline { 2 - 4 } & Close & 0.800 & 1.649 \\
\hline \multirow{3}{*}{ BPN } & High & 0.469 & 1.454 \\
\cline { 2 - 4 } & Low & 0.559 & 1.977 \\
\cline { 2 - 4 } & Close & 0.807 & 2.627 \\
\hline
\end{tabular}

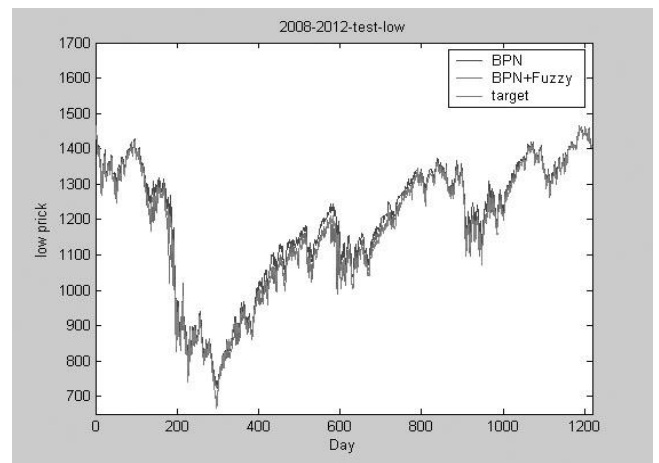

Fig. 8. 2008-2012 actual and predicted values comparison chart - the lowest price.

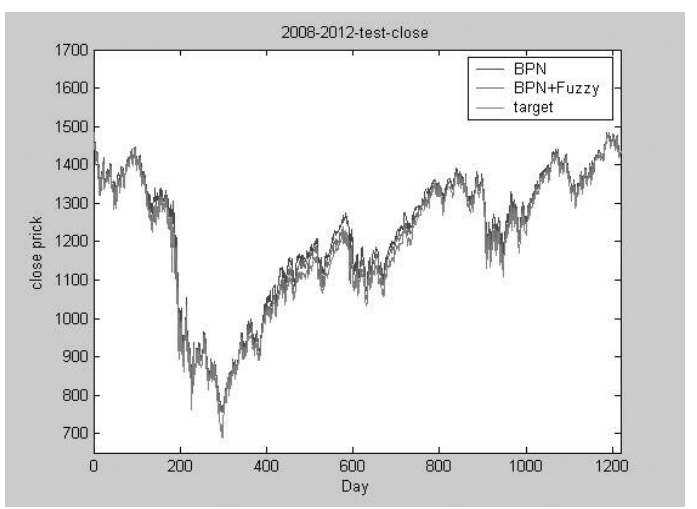

Fig. 9. 2008-2012 actual and predicted values comparison chart - the closing price.

\section{CONCLUSION AND FUTURE WORK}

This research brings up the idea of using the combination of BPNN and Fuzzy Controller as a new tool to predict the S\&P 500 Index, based on the Neural Network theory, Fuzzy Controller, and the stock price analysis.

According to the results, using the combination of BPNN and Fuzzy Controller can get a more precise prediction than the traditional Neural Network and BPNN.

Due to the Fuzzy Rules and different settings by different researchers, the results obtained from the Fuzzy Controller may be inaccurate. In the further research, researchers can discuss a way to establish a common setting for using Fuzzy Controller, in order to get a more accurate result.

\section{REFERENCES}

[1] I.-C. Yeh, Application of Artificial Neural Networks, Scholars Books Co., Ltd., 2002.

[2] I.-H. Chiu, "The Study of Neural Network to Predict Taiwan ETF-50 Stock Index Price," dissertation, Institute of information management, National Changhua University of Education, Changhua, Taiwan, 2005.

[3] S. Wei and X. Mian, "Stock Index Forecast with Back Propagation Neural Network Optimized by Genetic Algorithm," Second International Conference on Information and Computing Science, 2009.

[4] J.-D. Wang, Introduction and Application to Neural network and fuzzy control theory, Chuan Hwa Book CO., LTD, 2006.

[5] S. J. Chuang and C. Fyfe, "Power Load Prediction with Kernel Method," IEE, 2000.

[6] Option Trading Tips - Learn all About Trading Options, Sydney, Australia. (2005). [Online]. Available: www.optiontradingtips.com .

[7] C.-C. Kao, "Handwritten Signature Verification and the S\&P 500 Index Forecasting by Artificial Neural Network," National Kaohsiung Marine University, Electronic Communication Department, dissertation, May, 2012.

Shang-Jen Chuang was born in Taiwan. He received his computer and information system PhD degree from the University of Paisley, U.K. He is currently a associate professor, head of The Electronic Communication Engineering Department and director of computer center at the National Kaohsiung Marine University. His research interests include artificial neural network, database, programming and digital circuit.

Chung-Yung Lee was born in Taipei, Taiwan. He was graduated from the National Kaohsiung Marine University. He is currently a postgraduate in The Electronic Communication Engineering Department of National Kaohsiung Marine University. His research interests is artificial neural network. 